\title{
A class of solvable models in Condensed Matter Physics
}

\author{
F.Mancini \\ Dipartimento di Fisica "E.R. Caianiello" Unita' CNISM di Salerno, \\ Università degli Studi di Salerno, I-84081 Baronissi (SA), Italy
}

Received August 3, 2005

In this paper, we show that there is a large class of fermionic systems for which it is possible to find, for any dimension, a finite closed set of eigenoperators and eigenvalues of the Hamiltonian. Then, the hierarchy of the equations of motion closes and analytical expressions for the Green's functions are obtained in terms of a finite number of parameters, to be self-consistently determined. Several examples are given. In particular, for these examples it is shown that in the one-dimensional case it is possible to derive by means of algebraic constraints a set of equations which allow us to determine the self-consistent parameters and to obtain a complete exact solution.

Key words: strongly correlated electron systems, Hubbard model, Ising model, exact solution

PACS: $71.10 .-w, 71.10 . F d, 71.27 .+a$

One of the most intriguing problems in Condensed Matter Physics is the study of highly interacting fermionic systems [1]. The standard methods based on perturbation theories do not work well and the attention is paid to the development of alternative approximate formulations. In this context, it is interesting to cultivate the study of some solvable models, which are themselves of physical interest and which may furnish some indication on the solution of more complex models. In a recent paper [2], we have shown that there is a large class of fermionic systems for which it is possible to find a complete set of eigenoperators and eigenvalues of the Hamiltonian. Then, the hierarchy of the equations of motion closes and analytical exact expressions for the Green's functions (GF) can be obtained.

We consider a system of $q$ species of particles, satisfying the Fermi statistics and localized on the sites of a Bravais lattice. We suppose that the mass of the particles is very large and/or the interaction is so strong that the kinetic energy is negligible and the particles are frozen on the lattice sites. Let $c_{a}(i)$ and $c_{a}^{\dagger}(i)$ be the annihilation and creation operators of the particles of species $a$ in the Heisenberg picture: $i=(\mathbf{i}, t)$, where $\mathbf{i}$ stands for the lattice vector $R_{\mathbf{i}}$. These operators satisfy the canonical anti-commutation relations

$$
\begin{aligned}
& \left\{c_{a}(\mathbf{i}, t), c_{b}^{\dagger}(\mathbf{j}, t)\right\}=\delta_{a b} \delta_{\mathbf{i j}}, \\
& \left\{c_{a}(\mathbf{i}, t), c_{b}(\mathbf{j}, t)\right\}=\left\{c_{a}^{\dagger}(\mathbf{i}, t), c_{b}^{\dagger}(\mathbf{j}, t)\right\}=0 .
\end{aligned}
$$

A quite general Hamiltonian for these systems reads as

$$
\begin{aligned}
H= & \sum_{\mathbf{i} a} V_{a}(\mathbf{i}) n_{a}(i)+\frac{1}{2} \sum_{\mathbf{i} \mathbf{j}} \sum_{a b} V_{a b}(\mathbf{i}, \mathbf{j}) n_{a}(i) n_{b}(j) \\
& +\frac{1}{3 !} \sum_{\mathbf{i} \mathbf{j} \mathbf{l}} \sum_{a b c} V_{a b c}(\mathbf{i}, \mathbf{j}, \mathbf{l}) n_{a}(i) n_{b}(j) n_{c}(l)+\cdots,
\end{aligned}
$$

where $V_{a}(\mathbf{i})$ represents an external field acting on the particle $a ; n_{a}(i)=c_{a}^{\dagger}(i) c_{a}(i)$ is the particle density operator of the species $a ; V_{a b}, V_{a b c}, \cdots$ are the two-body, three-body, $\ldots$ potentials. In general, the potentials will be translationally invariant and will depend only on the differences between the coordinates of the particles. 
The class of systems described by the Hamiltonian (2) is very large and of direct physical interest. By appropriate choice of the potentials it may describe multiband electronic systems dominated by charge correlations and by magnetic interactions in the $s_{z}$-channel, where $s_{z}$ is the third component of the spin. Furthermore, let $n(i)=\sum_{a} c_{a}^{\dagger}(i) c_{a}(i)$ be the total particle density and consider the transformation $n(i)=q / 2+S(i)$. Under this transformation the Hamiltonian $(2)$ is mapped to spin- $q / 2$ Ising-like models.

Now, if the interaction potentials have a finite range, the model Hamiltonian is always solvable. The proof of this statement is as follows. Firstly, it is immediate to see that the density operator $n_{a}(i)$ does not depend on time

$$
\mathrm{i} \frac{\partial}{\partial t} n_{a}(i)=\left[n_{a}(i), H\right]=0 .
$$

Then, in order to use the equations of motion and GF formalism we must start from the Heisenberg equation for the fermionic field. It is immediate to see that $c_{a}(i)$ satisfies the equation of motion

$$
\mathrm{i} \frac{\partial}{\partial t} c_{a}(i)=V_{a}(\mathbf{i}) c_{a}(i)+\sum_{\mathbf{j} b} V_{a b}(\mathbf{i}, \mathbf{j}) n_{b}(j) c_{a}(i)+\frac{1}{2} \sum_{\mathbf{j} \mathbf{l}} \sum_{b c} V_{a b c}(\mathbf{i}, \mathbf{j}, \mathbf{l}) n_{b}(j) n_{c}(l) c_{a}(i)+\cdots
$$

The dynamics has generated other field operators of higher complexity. By taking time derivatives of increasing order, more and more complex operators are generated. These operators might be named composite operators, as they are all expressed in terms of the original fields $c_{a}(i)$. Due to the finite range of the interaction, the higher-order field operators generated by the dynamics extend over a finite number of sites. Then, since the particle operator satisfies the algebra $\left[n_{a}(i)\right]^{p}=n_{a}(i)$ (for $p \geqslant 1$ ), the number of composite operators is finite and a complete set of eigenoperators of the Hamiltonian can be found. If $n$ is the number of independent composite operators, we can construct a $n$-multiplet operator

$$
\psi(i)=\left(\begin{array}{c}
\psi_{1}(i) \\
\psi_{2}(i) \\
\vdots \\
\psi_{n}(i)
\end{array}\right)
$$

which satisfies the Heisenberg equation

$$
\mathrm{i} \frac{\partial}{\partial t} \psi(i)=[\psi(i), H]=\epsilon \psi(i)
$$

where the $n \times n$ matrix $\epsilon$ will be denominated as the energy matrix. Note that we are using a vectorial notation: the field $\psi_{m}(i)$ is itself a multiplet of rank $q$. Once the composite operator and the energy matrix have been determined, an exact solution of the Hamiltonian can be formally obtained. Let us define the retarded Green's function

$$
G(i, j)=\left\langle R\left[\psi(i) \psi^{\dagger}(j)\right]\right\rangle=\theta\left(t_{i}-t_{j}\right)\left\langle\left\{\psi(i), \psi^{\dagger}(j)\right\}\right\rangle
$$

where $\langle\cdots\rangle$ denotes the quantum-statistical average over the grand canonical ensemble. By means of the Heisenberg equation (6), we obtain in momentum space the equation

$$
[\omega-\epsilon] G(\mathbf{k}, \omega)=I(\mathbf{k}),
$$

where $I(\mathbf{k})$ is the Fourier transform of the normalization matrix, defined as

$$
I(\mathbf{i}, \mathbf{j})=\left\langle\left\{\psi(\mathbf{i}, t), \psi^{\dagger}(\mathbf{j}, t)\right\}\right\rangle .
$$

The solution of equation (8) is

$$
G(\mathbf{k}, \omega)=\sum_{m=1}^{n} \frac{\sigma^{(m)}(\mathbf{k})}{\omega-E_{m}+\mathrm{i} \delta}
$$


where $E_{m}$ are the eigenvalues of the energy matrix $\epsilon$. The spectral density matrices $\sigma^{(m)}(\mathbf{k})$ are calculated by means of the formula [3]

$$
\sigma_{\alpha \beta}^{(m)}(\mathbf{k})=\Omega_{\alpha m} \sum_{\gamma}\left[\Omega_{m \gamma}\right]^{-1} I_{\gamma \beta}(\mathbf{k})
$$

where $\Omega$ is the matrix whose columns are the eigenvectors of the matrix $\epsilon$. The correlation function $C(i, j)=\left\langle\psi(i) \psi^{\dagger}(j)\right\rangle$ can be immediately calculated from (10) and one obtains

$$
\begin{aligned}
C(i, j) & =\frac{1}{N} \sum_{\mathbf{k}} \frac{1}{2 \pi} \int \mathrm{d} \omega \mathrm{e}^{\mathrm{i} \mathbf{k} \cdot\left(\mathbf{R}_{i}-\mathbf{R}_{j}\right)-\mathrm{i} E_{m}\left(t_{i}-t_{j}\right)} C(\mathbf{k}, \omega) \\
C(\mathbf{k}, \omega) & =\pi \sum_{m=1}^{n} \delta\left(\omega-E_{m}\right) T_{m} \sigma^{(m)}(\mathbf{k})
\end{aligned}
$$

with $T_{m}=1+\tanh \left(\beta E_{m} / 2\right), \beta$ is the inverse temperature. By similar technique we can easily calculate [4] multi-point correlation functions as $C\left(i, j ; l_{1}, l_{2}, \cdots l_{s}\right)=\left\langle\psi(i) \psi^{\dagger}(j) n\left(l_{1}\right) n\left(l_{2}\right) \cdots n\left(l_{s}\right)\right\rangle$.

Equations (10) and (12) are an exact solution of the model Hamiltonian (2). However, the knowledge of the GF is not fully achieved yet. The algebra of the field $\psi(i)$ is not canonical: as a consequence, the normalization matrix $I(\mathbf{k})$ in the equation of motion (8) contains some unknown static correlation functions. Generally, these correlators are expectation values of operators not belonging to the chosen basis and should be self-consistently calculated. According to the scheme of calculations proposed by the composite operator method [3,5], one way of calculating the unknown correlators is by specifying the representation where the GF are realized. The knowledge of the Hamiltonian and of the operatorial algebra is not sufficient to completely determine the GF. The GF refer to a specific representation (i.e., to a specific choice of the Hilbert space) and this information must be supplied to the equations of motion that alone are not sufficient to completely determine the GF. The procedure is as follows. From the algebra it is possible to derive several relations among the operators. We shall call algebra constraints (AC) all possible relations among the operators dictated by the algebra. This set of relations valid at microscopic level must be also satisfied at macroscopic level, when expectation values are considered. These considerations lead to some self-consistent equations which will be used to fix the unknown correlators appearing in the normalization matrix. An immediate set of rules is given by the equation

$$
\left\langle\psi(i) \psi^{\dagger}(i)\right\rangle=\frac{1}{N} \sum_{\mathbf{k}} \frac{1}{2 \pi} \int \mathrm{d} \omega C(\mathbf{k}, \omega),
$$

where the l.h.s. is fixed by the AC and by the boundary conditions compatible with the phase under investigation, while in the r.h.s. the correlation function $C(\mathbf{k}, \omega)$ is computed by means of the expression (12). The use of (13) will lead to a set of exact relations among the correlation functions. Unfortunately, for the class of systems considered, the number of equations is always less than the number of correlation functions and extra equations are needed. To this end we have recently found $[2,4]$ a set of $\mathrm{AC}$ capable of giving other relations among the correlation functions. Let us suppose that there exist some operators, $O$, which project out of the Hamiltonian a reduced part

$$
\mathrm{OH}=\mathrm{OH}_{0}
$$

When $H_{0}$ and $H_{I}=H-H_{0}$ commute, an important rule which descends from the AC (14) is that the quantum statistical averages over the complete Hamiltonian $H$ must coincide with the average over the reduced Hamiltonian $H_{0}$

$$
\operatorname{Tr}\left\{O \mathrm{e}^{-\beta H}\right\}=\operatorname{Tr}\left\{O \mathrm{e}^{-\beta H_{0}}\right\} .
$$

For a variety of one-dimensional models, we have found that the use of the condition (15) allows us to close the set of equations for the correlation functions and to obtain an exact solution of the 
model. For dimensions higher than one these conditions are not sufficient and more equations are necessary.

We now apply the above procedure to some specific models, obtained by special choice of the interaction potentials. We shall consider a $d$-dimensional hypercubic Bravais lattice.

\section{First example}

We consider a $q$-state model described by the Hamiltonian

$$
H=-\mu \sum_{\mathbf{i}} n(i)+\mathrm{d} V \sum_{\mathbf{i}} n(i) n^{\alpha}(i)
$$

where $\mu$ is the chemical potential, $n(i)=\sum_{a} n_{a}(i)$ is the total particle density. Hereafter, for a generic operator $\Phi(i)$ we use the notation $\Phi^{\alpha}(i)=\sum_{\mathbf{j}} \alpha_{\mathbf{i j}} \Phi(\mathbf{j}, t)$, where $\alpha_{\mathbf{i j}}$ is the projector on the first-nearest neighbor sites. This model might describe a system of $q$-electron bands, interacting through an intersite Coulomb potential of strength $V$. Alternatively, by means of the transformation $n(i)=q / 2+S(i)$ the Hamiltonian (16) takes the form

$$
H=-h \sum_{\mathbf{i}} S(i)-J \sum_{\mathbf{i}} S(i) S^{\alpha}(i)+E_{0}
$$

where $J=-\mathrm{d} V, h=\mu-q \mathrm{~d} V, E_{0}=q / 2(-\mu+q / 2 \cdot \mathrm{d} V) N ; N$ is the number of sites. Hamiltonian (17) is just the spin- $q / 2$ Ising model with nearest neighbor interactions in the presence of an external magnetic field $h$. The following recursion rule can be established [4] for the operator $\left[n^{\alpha}(i)\right]^{p}$

$$
\left[n^{\alpha}(i)\right]^{p}=\sum_{m=1}^{2 q d} A_{m}^{(p)}\left[n^{\alpha}(i)\right]^{m}
$$

where the coefficients $A_{m}^{(p)}$ are rational numbers, satisfying the relation $\sum_{m=1}^{2 q d} A_{m}^{(p)}=1$, that can be easily determined by the algebra and by the structure of the lattice [4]. Owing to this rule, the set of eigenoperators of the Hamiltonian is given by

$$
\psi(i)=\left(\begin{array}{c}
c(i) \\
c(i) n^{\alpha}(i) \\
\vdots \\
c(i)\left[n^{\alpha}(i)\right]^{2 q d}
\end{array}\right), \quad[\psi(i), H]=\epsilon \psi(i),
$$

where the energy matrix $\epsilon$ can be calculated by means of the equation of motion and the recursion rule (18). The eigenvalues $E_{m}$ of $\epsilon$ are given by

$$
E_{m}=-\mu+(m-1) V, \quad(m=1,2, \cdots 2 q d+1) .
$$

The retarded Green's functions and the correlation functions can be exactly calculated by applying the scheme of calculations illustrated above. Then, by using the AC (15) it is possible to derive the self-consistent equations

$$
\kappa^{(k-1)}-\lambda^{(k-1)}=\frac{1}{2} \sum_{m=1}^{2 q d+1} T_{m} \sigma_{1, k}^{(m)}, \quad(k=1, \ldots, 2 q d+1)
$$

where $T_{m}=1+\tanh \left(\beta E_{m} / 2\right)$ and $\sigma^{(m)}$ are the spectral density matrices, that can be calculated by means of (11) and are expressed in terms of the quantities $\kappa^{(p)}$. Equations (21) give a set of exact relations, valid for any value of $q$ and $d$, among the correlation functions

$$
\begin{aligned}
\kappa^{(p)} & =\left\langle\left[n^{\alpha}(i)\right]^{p}\right\rangle, \\
\lambda^{(p)} & =\left\langle n(i)\left[n^{\alpha}(i)\right]^{p}\right\rangle .
\end{aligned} \quad(p=0,1, \cdots 2 q d),
$$


Unfortunately, the number of equations is not sufficient and we need other conditions. We have studied the cases of $q=1,2,3$ in $[4,6,7]$, respectively. In all these cases for one-dimensional systems, it is possible to find by means of the algebraic constraints (15) the necessary equations to close the set. These extra conditions are obtained by exploiting the following algebraic conditions. Let us divide the Hamiltonian as

$$
H=H_{0}+2 V n(i) n^{\alpha}(i)
$$

where, due to translational invariance, $\mathbf{i}$ is a generic site of the infinite chain. Then, we have

$$
\begin{aligned}
& q=1, c^{\dagger}(i) \mathrm{e}^{-\beta H}=c^{\dagger}(i) \mathrm{e}^{-\beta H_{0}}, \\
& q=2, \quad \xi^{\dagger}(i) \mathrm{e}^{-\beta H}=\xi^{\dagger}(i) \mathrm{e}^{-\beta H_{0}}, \\
& \eta^{\dagger}(i) \mathrm{e}^{-\beta H}=\eta^{\dagger}(i)\left\{1+\sum_{m=1}^{4} f_{m}\left[n^{\alpha}(i)\right]^{m}\right\} \mathrm{e}^{-\beta H_{0}}, \\
& \xi^{\dagger}(i) \mathrm{e}^{-\beta H}=\xi^{\dagger}(i) \mathrm{e}^{-\beta H_{0}}, \\
& \eta^{\dagger}(i) \mathrm{e}^{-\beta H}=\eta^{\dagger}(i)\left\{1+\sum_{m=1}^{6} f_{m}\left[n^{\alpha}(i)\right]^{m}\right\} \mathrm{e}^{-\beta H_{0}}, \\
& \zeta^{\dagger}(i) \mathrm{e}^{-\beta H}=\zeta^{\dagger}(i)\left\{1+\sum_{m=1}^{6}\left(2 f_{m}+g_{m}\right)\left[n^{\alpha}(i)\right]^{m}\right\} \mathrm{e}^{-\beta H_{0}} .
\end{aligned}
$$

For $q=2$ the definitions are:

$$
\xi_{a}(i)=[1-n(i)] c_{a}(i), \quad \eta_{a}(i)=n(i) c_{a}(i)
$$

The operators $\xi_{a}(i)$ and $\eta_{a}(i)$ induce the transitions $0 \Leftrightarrow 1,1 \Leftrightarrow 2$, respectively. For $q=3$ the definitions are:

$$
\xi_{a}(i)=[1-n(i)+D(i)] c_{a}(i), \quad \eta_{a}(i)=[n(i)-2 D(i)] c_{a}(i), \quad \zeta_{a}(i)=D(i) c_{a}(i),
$$

where $D(i)$ is the double occupancy operator, defined as

$$
D(i)=n_{1}(i) n_{2}(i)+n_{1}(i) n_{3}(i)+n_{2}(i) n_{3}(i) .
$$

The projection operators $\xi_{a}, \eta_{a}$ and $\zeta_{a}$ induce the transitions among states with different particle numbers: $0 \Leftrightarrow 1,1 \Leftrightarrow 2,2 \Leftrightarrow 3$, respectively. The quantities $f_{m}$ and $g_{m}$ are the known functions of $\beta V$.

\section{Second example}

Let us consider two species of particles, say $a$ and $b$, and consider the Hamiltonian

$$
H=-\mu \sum_{\mathbf{i}} n(i)+U \sum_{\mathbf{i}} D(i)+\mathrm{d} V \sum_{\mathbf{i}} n(i) n^{\alpha}(i),
$$

where $n(i)=n_{a}(i)+n_{b}(i)$ and $D(i)=n_{a}(i) n_{b}(i)$ are the total particle density and double occupancy operators, respectively. This Hamiltonian is just the extended Hubbard model in the ionic limit, where $U$ and $V$ are the on-site and inter-site Coulomb interactions, respectively. The two species of particles, $a$ and $b$, are in this case the electrons with spin up and down, respectively. By means of the transformation $n(i)=1+S(i),(30)$ can be cast in the form

$$
H=-h \sum_{\mathbf{i}} S(i)+\Delta \sum_{\mathbf{i}} S^{2}(i)-J \sum_{\mathbf{i}} S(i) S^{\alpha}(i)+E_{0},
$$


where $J=-\mathrm{d} V, h=\mu-2 \mathrm{~d} V-U / 2, \Delta=U / 2, E_{0}=(-\mu+\mathrm{d} V) N$. Hamiltonian (31) is just the Ising spin-1 model with nearest-neighbor interactions in the presence of a crystal field $\Delta$ and an external magnetic field $h$ [8-11]. We now define the composite operators

$$
\psi^{(\xi)}(i)=\left(\begin{array}{c}
\xi(i) \\
\xi(i) n^{\alpha}(i) \\
\vdots \\
\xi(i)\left[n^{\alpha}(i)\right]^{4 d}
\end{array}\right), \quad \psi^{(\eta)}(i)=\left(\begin{array}{c}
\eta(i) \\
\eta(i) n^{\alpha}(i) \\
\vdots \\
\eta(i)\left[n^{\alpha}(i)\right]^{4 d}
\end{array}\right)
$$

where $\xi(i)$ and $\eta(i)$ are the Hubbard operators, defined in (27). By means of (18), these fields are eigenoperators of the Hamiltonian (30)

$$
\mathrm{i} \frac{\partial}{\partial t} \psi^{(\xi)}(i)=\left[\psi^{(\xi)}(i), H\right]=\epsilon^{(\xi)} \psi^{(\xi)}(i), \quad \mathrm{i} \frac{\partial}{\partial t} \psi^{(\eta)}(i)=\left[\psi^{(\eta)}(i), H\right]=\epsilon^{(\eta)} \psi^{(\eta)}(i),
$$

where $\epsilon^{(\xi)}$ and $\epsilon^{(\eta)}$ are the energy matrices, of rank $(4 d+1) \times(4 d+1)$, which can be calculated by means of the equations of motion and the recursion rule (18). The eigenvalues of the energy matrices are given by

$$
E_{m}^{(\xi)}=-\mu+(m-1) V, \quad E_{m}^{(\eta)}=-\mu+U+(m-1) V, \quad(m=1,2, \cdots 4 q d+1) .
$$

The retarded and the correlation functions can be exactly calculated by applying the scheme of calculations illustrated above. Then, by using the AC (13) it is possible to derive the self-consistent equations

$$
\kappa^{(k-1)}-\frac{1}{2} \lambda^{(k-1)}=\frac{1}{2} \sum_{m=1}^{4 d+1}\left[T_{m}^{(\xi)} \sigma_{1, k}^{(\xi, m)}+T_{m}^{(\eta)} \sigma_{1, k}^{(\eta, m)}\right], \quad(k=1, \cdots 4 d+1),
$$

where $T_{m}^{(a)}=1+\tanh \left(\beta E_{m}^{(a)} / 2\right)$ with $a=\xi, \eta \cdot \sigma^{(a, m)}$ are the spectral density matrices, that can be calculated by means of (11) and are expressed in terms of the quantities $\kappa^{(p)}$ and $\lambda^{(p)}$. Equations (35) give a set of exact relations, valid for any dimension, among the correlation functions (22). Unfortunately, the number of equations is not sufficient and we need other conditions. Our study [6] has shown that for the one-dimensional case, it is possible to find by means of the algebraic constraints (15) the necessary equations to close the set (35). These extra conditions are obtained by exploiting the following algebraic conditions

$$
\begin{aligned}
& \xi^{\dagger}(i) \mathrm{e}^{-\beta H}=\xi^{\dagger}(i) \mathrm{e}^{-\beta H_{0}} \\
& D(i) \mathrm{e}^{-\beta H}=D(i)\left\{1+\sum_{m=1}^{4}\left(2 f_{m}+g_{m}\right)\left[n^{\alpha}(i)\right]^{m}\right\} \mathrm{e}^{-\beta H_{0}}
\end{aligned}
$$

where $H_{0}=H-2 V n(i) n^{\alpha}(i)$.

\section{Third example}

The model (30) can be generalized by considering 3- and 4-body potentials. In particular, by considering one-dimensional systems, let us take

$$
\begin{aligned}
H= & -\mu \sum_{\mathbf{i}} n(i)-\gamma \sum_{\mathbf{i}} D(i)+V \sum_{\mathbf{i}} n(i) n^{\alpha}(i) \\
& +U \sum_{\mathbf{i}} D(i) D^{\alpha}(i)-U \sum_{\mathbf{i}} D(i) n^{\alpha}(i)
\end{aligned}
$$

This Hamiltonian can be mapped to the following model

$$
H=-h \sum_{\mathbf{i}} S(i)+\Delta \sum_{\mathbf{i}} S^{2}(i)-J \sum_{\mathbf{i}} S(i) S^{\alpha}(i)-K \sum_{\mathbf{i}} S^{2}(i) S^{2 \alpha}(i)+E_{0},
$$


where $J=U / 4-V, K=-U / 4, h=\mu+\gamma / 2+U / 2-2 V, \Delta=-U / 2-\gamma / 2, E_{0}=(-\mu+V) N$. This Hamiltonian is just the one-dimensional Blume-Emery-Griffiths model [12]. We now define the composite operators

$$
\psi^{(\xi)}(i)=\left(\begin{array}{c}
\xi(i) \\
\xi(i) n^{\alpha}(i) \\
\xi(i)\left[n^{\alpha}(i)\right]^{2} \\
\xi(i)\left[n^{\alpha}(i)\right]^{3} \\
\xi(i)\left[n^{\alpha}(i)\right]^{4} \\
\xi(i) D^{\alpha}(i) \\
\xi(i)\left[D^{\alpha}(i)\right]^{2}
\end{array}\right), \quad \psi^{(\eta)}(i)=\left(\begin{array}{c}
\eta(i) \\
\eta(i) n^{\alpha}(i) \\
\eta(i)\left[n^{\alpha}(i)\right]^{2} \\
\eta(i)\left[n^{\alpha}(i)\right]^{3} \\
\eta(i)\left[n^{\alpha}(i)\right]^{4} \\
\eta(i) D^{\alpha}(i) \\
\eta(i)\left[D^{\alpha}(i)\right]^{2}
\end{array}\right)
$$

where $\xi(i)$ and $\eta(i)$ are the Hubbard operators, [cf. (27)]. These fields are eigenoperators of the Hamiltonian (37). The corresponding eigenvalues are

$$
E_{m}^{(\xi)}=\left(\begin{array}{c}
-\mu \\
-\mu+V \\
-\mu+2 V \\
-\mu-U+2 V \\
-\mu-\frac{1}{2} U+2 V \\
-\mu-U+4 V \\
-\mu-\frac{1}{2} U+3 V
\end{array}\right), \quad E_{m}^{(\eta)}=\left(\begin{array}{c}
-\mu-\gamma \\
-\mu-\gamma-\frac{1}{2} U+V \\
-\mu-\gamma-U+2 V \\
-\mu-\gamma+2 V \\
-\mu-\gamma-\frac{1}{2} U+2 V \\
-\mu-\gamma-U+4 V \\
-\mu-\gamma-U+3 V
\end{array}\right) .
$$

The retarded and the correlation functions can be exactly calculated by applying the scheme of calculations illustrated above. Then, by using the AC (13) it is possible to derive [13] the selfconsistent equations

$$
\begin{aligned}
\lambda^{(0)}-\delta^{(1)} & =\frac{1}{2} \sum_{m=1}^{7} T_{m}^{(\eta)} \sigma_{1,1}^{(\eta, m)}, \\
\kappa^{(k-1)}-\frac{1}{2} \lambda^{(k-1)} & =\frac{1}{2} \sum_{m=1}^{7}\left[T_{m}^{(\xi)} \sigma_{1, k}^{(\xi, m)}+T_{m}^{(\eta)} \sigma_{1, k}^{(\eta, m)}\right], \quad(k=1, \cdots 5), \\
\delta^{(k-5)}-\frac{1}{2} \theta^{(k-5)} & =\frac{1}{2} \sum_{m=1}^{7}\left[T_{m}^{(\xi)} \sigma_{1, k}^{(\xi, m)}+T_{m}^{(\eta)} \sigma_{1, k}^{(\eta, m)}\right], \quad(k=6,7) .
\end{aligned}
$$

where the definitions are the same as in example 2, and the new correlation functions are defined as

$$
\begin{aligned}
& \delta^{(p)}=\left\langle\left[D^{\alpha}(i)\right]^{p}\right\rangle \\
& \theta^{(p)}=\left\langle n(i)\left[D^{\alpha}(i)\right]^{p}\right\rangle, \quad(p=1,2) .
\end{aligned}
$$

$\sigma^{(a, m)}$ are the spectral density matrices and are expressed in terms of the quantities $\kappa^{(p)}, \lambda^{(p)}, \delta^{(p)}$, $\theta^{(p)}$. Equations (41) give a set of exact relations among the correlation functions (22) and (42). By exploiting the algebraic condition $\xi^{\dagger}(i) \mathrm{e}^{-\beta H}=\xi^{\dagger}(i) \mathrm{e}^{-\beta H_{0}}$ it is possible to derive [13] the self-consistent equations

$$
\begin{aligned}
C_{1,3}^{(\xi)} & =C_{1,1}^{(\xi)}\left[\frac{1}{2} X_{1}+X_{2}+\frac{1}{2} X_{1}^{2}\right], \\
C_{1,4}^{(\xi)} & =C_{1,1}^{(\xi)}\left[\frac{1}{4} X_{1}+\frac{3}{2} X_{2}+\frac{3}{2} X_{1} X_{2}+\frac{3}{4} X_{1}^{2}\right], \\
C_{1,5}^{(\xi)} & =C_{1,1}^{(\xi)}\left[\frac{1}{8} X_{1}+\frac{7}{4} X_{2}+\frac{9}{2} X_{1} X_{2}+\frac{7}{8} X_{1}^{2}+\frac{3}{2} X_{2}^{2}\right] \\
C_{1,7}^{(\xi)} & =C_{1,1}^{(\xi)}\left[\frac{1}{2} X_{2}+\frac{1}{2} X_{2}^{2}\right]
\end{aligned}
$$


where

$$
X_{1}=\frac{C_{1,2}^{(\xi)}}{C_{1,1}^{(\xi)}}, \quad X_{2}=\frac{C_{1,6}^{(\xi)}}{C_{1,1}^{(\xi)}}
$$

$C_{1, k}^{(\xi)}$ is an equal time correlation function $C_{1, k}^{(\xi)}=\left\langle\psi_{1}^{(\xi)}(i) \psi_{1}^{(\xi) \dagger}(i)\right\rangle$, expressed in terms of the parameters $\kappa^{(p)}, \lambda^{(p)}, \delta^{(p)}, \theta^{(p)}$ by means of the relation

$$
C_{1, k}^{(\xi)}=\frac{1}{2} \sum_{m=1}^{7} T_{m}^{(\xi)} \sigma_{1, k}^{(\xi, m)} .
$$

\section{Fourth example}

Let us consider the case of two particles, characterized by spin $\sigma=\uparrow, \downarrow(+,-)$, and the choice the potentials as

$$
V_{\sigma}(\mathbf{i})=-\mu-\sigma h, \quad V_{\sigma, \sigma^{\prime}}(\mathbf{i}, \mathbf{j})=2 V d\left(2 \delta_{\sigma, \sigma^{\prime}}-1\right) \alpha_{\mathbf{i}, \mathbf{j}},
$$

$h$ is the intensity of the external magnetic field. The Hamiltonian is

$$
H=-\mu \sum_{\mathbf{i}} n(i)+\mathrm{d} V \sum_{\mathbf{i}} n_{3}(i) n_{3}^{\alpha}(i)-h \sum_{\mathbf{i}} n_{3}(i)
$$

where $n_{3}(i)=n_{\uparrow}(i)-n_{\downarrow}(i)$ is the third component of the spin density operator. Let us restrict the analysis to one-dimensional systems. The following recursion rule can be established for the operator $\left[n_{3}^{\alpha}(i)\right]^{p}$

$$
\left[n_{3}^{\alpha}(i)\right]^{p}=\sum_{m=1}^{4} A_{m}^{(p)}\left[n_{3}^{\alpha}(i)\right]^{m}
$$

where the coefficients $A_{m}^{(p)}$ are rational numbers, satisfying the relation $\sum_{m=1}^{4} A_{m}^{(p)}=1$, that can be easily determined by the algebra and the structure of the lattice. Owing to this rule, the set of eigenoperators of the Hamiltonian (47) is given by

$$
\begin{gathered}
\psi(i)=\left(\begin{array}{l}
\psi_{\uparrow}(i) \\
\psi_{\downarrow}(i)
\end{array}\right), \quad \psi_{\sigma}(i)=\left(\begin{array}{l}
c_{\sigma}(i) \\
c_{\sigma}(i) n_{3}^{\alpha}(i) \\
c_{\sigma}(i)\left[n_{3}^{\alpha}(i)\right]^{2} \\
c_{\sigma}(i)\left[n_{3}^{\alpha}(i)\right]^{3} \\
c_{\sigma}(i)\left[n_{3}^{\alpha}(i)\right]^{4}
\end{array}\right), \\
\mathrm{i} \frac{\partial}{\partial t} \psi_{\sigma}(i)=\epsilon^{(\sigma)} \psi_{\sigma}(i)
\end{gathered}
$$

The energy matrix $\epsilon^{(\sigma)}$ is a $5 \times 5$ matrix with the following expression

$$
\epsilon^{(\sigma)}=\left(\begin{array}{ccccc}
-\mu-\sigma h & 2 \sigma V & 0 & 0 & 0 \\
0 & -\mu-\sigma h & 2 \sigma V & 0 & 0 \\
0 & 0 & -\mu-\sigma h & 2 \sigma V & 0 \\
0 & 0 & 0 & -\mu-\sigma h & 2 \sigma V \\
0 & -\frac{1}{2} \sigma V & 0 & \frac{5}{2} \sigma V & -\mu-\sigma h
\end{array}\right) .
$$

The eigenvalues of the matrix $\epsilon^{(\sigma)}$ are

$$
E_{m}^{(\sigma)}=\left(\begin{array}{c}
-\mu-\sigma h \\
-\mu-\sigma h-2 V \\
-\mu-\sigma h-V \\
-\mu-\sigma h+V \\
-\mu-\sigma h+2 V
\end{array}\right)
$$


The retarded Green's functions and the correlation functions can be exactly calculated by applying the scheme of calculations illustrated above. Then, by using the AC (13) it is possible to derive the self-consistent equations

$$
\begin{aligned}
2 \kappa^{(k-1)}-\lambda^{(k-1)} & =\frac{1}{2} \sum_{m=1}^{5}\left[T_{m}^{(\uparrow)} \sigma_{1, k}^{(\uparrow, m)}+T_{m}^{(\downarrow)} \sigma_{1, k}^{(\downarrow, m)}\right], \\
\theta^{(k-1)} & =\frac{1}{2} \sum_{m=1}^{5}\left[T_{m}^{(\uparrow)} \sigma_{1, k}^{(\uparrow, m)}-T_{m}^{(\downarrow)} \sigma_{1, k}^{(\downarrow, m)}\right], \quad(k=1, \cdots 5),
\end{aligned}
$$

where $T_{m}^{(\sigma)}=1+\tanh \left(\beta E_{m}^{(\sigma)} / 2\right)$ and $\sigma^{(\sigma, m)}$ are the spectral density matrices, that can be calculated by means of (11)and are expressed in terms of the quantities $\kappa^{(p)}$. Equations (53) give a set of exact relations among the correlation functions

$$
\begin{aligned}
\kappa^{(p)} & =\left\langle\left[n_{3}^{\alpha}(i)\right]^{p}\right\rangle \\
\lambda^{(p)} & =\left\langle n(i)\left[n_{3}^{\alpha}(i)\right]^{p}\right\rangle, \\
\theta^{(p)} & =\left\langle n_{3}(i)\left[n_{3}^{\alpha}(i)\right]^{p}\right\rangle,
\end{aligned}
$$

For this model we can also use the AC (15) in order to derive extra equations capable of closing the set (53) for the correlation functions. Details will be presented elsewhere.

Summarizing, we have shown that a large class of systems of localized particles, satisfying Fermi statistics and subject to finite-range interactions, is always solvable, in the sense that a complete finite set of eigenoperators and eigenvalues of the Hamiltonian can be found. This knowledge allows us to derive analytical expressions for the Green's functions and for the correlation functions and a set of exact relations among the correlation functions can be derived. As an illustration we have considered several examples. In all the studied models we have shown that for the case of one dimension it is possible to use algebraic constraints which permit to close the set of self-consistent equations and to obtain exact solutions. For higher dimensions more self-consistent equations are needed. This problem is now under investigation.

\section{References}

1. Fulde P. Electron Correlations in Molecules and Solids. Springer-Verlag, Berlin, 1995.

2. Mancini F., Europhys. Lett., 2005, 70, 485.

3. Mancini F., Avella A., Eur. Phys. J. B, 2003, 36, 37.

4. Mancini F., Eur. Phys. J. B, 2005, 45, 497.

5. Mancini F., Avella A., Adv. Phys., 2004, 53, 537.

6. Mancini F., Eur. Phys. J. B, 2005, 47, 527.

7. Avella A., Mancini F., Eur. Phys. J. B, 2006, 50, 527.

8. Blume M., Phys. Rev., 1966, 141, 517.

9. Capel H., Physica, 1966, 32, 966.

10. Capel H., Physica, 1967, 33, 295.

11. Capel H., Physica, 1967, 37, 423.

12. Blume M., Emery V., Griffiths R., Phys. Rev. A, 1971, 4, 1071.

13. Mancini F., Mancini F.P., in preparation, 2005. 


\section{Клас розв'язуваних моделей у фізиці конденсованого стану}

Ф.Манчіні

Університет Салерно, фізичний факультет, 84081 Бароніссі, Салерно, Італія

Отримано 3 серпня 2005 р.

В цій статті ми показуємо, що існує великий клас ферміонних систем, для яких можна знайти, для довільної вимірності, скінченний замкнутий набір власних операторів і власних значень гамільтоніану. Тоді, ієрархія рівнянь руху є замкнутою і вирази для функцій Гріна отримуються в просторі скінченного числа параметрів, що визначаються самоузгоджено. Наводиться декілька прикладів, для яких, зокрема, показано, що в одновимірному випадку за допомогою алгебраїчних в'язей можна отримати набір рівнянь, які дозволяють визначити самоузгоджені параметри та знайти повний точний розв'язок.

Ключові слова: сильно скорельовані електронні системи, модель Габбарда, модель Ізинга, точний розв'язок

PACS: 71.10.-w, 71.10.Fd, 71.27.+a 